\title{
Faixas de suficiência para a cultura do algodão no centro-oeste do Brasil: I. Macronutrientes
}

\author{
Sufficiency range for cotton cropped in Brazil midwest: I. Macronutrients
}

\author{
Marcos Antonio Camacho ${ }^{\mathrm{I}}$ William Natale ${ }^{\mathrm{II}}$ José Carlos Barbosa ${ }^{\mathrm{II}}$
}

\section{RESUMO}

\begin{abstract}
A cultura do algodoeiro possui informações para a diagnose foliar estabelecidas para o Brasil, entretanto, esses valores de referência foram obtidos em sistemas produtivos distintos dos atualmente praticados no país, necessitando atualização destas referências. $O$ trabalho objetivou estabelecer faixas de suficiência para macronutrientes na cultura do algodoeiro por meio do método da chance matemática. $O$ trabalho foi realizado no período de 2000 a 2003 nos municípios de Costa Rica (MS), Diamantino e Sapezal (MT), totalizando 152 amostras de tecido vegetal (folha completa na quinta posição da haste principal, coletada no início do florescimento) e produtividade ( $\mathrm{em} \mathrm{kg} \mathrm{ha}^{-1}$ de algodão em caroço) de lavouras comerciais de algodão. Foram determinados os teores de macronutrientes ( $\mathrm{N}, \mathrm{P}, \mathrm{K}, \mathrm{Ca}, \mathrm{Mg}$ e $S)$ e os dados foram submetidos ao método da chance matemática, utilizando-se de produtividades diferentes (4.000 e $4.500 \mathrm{~kg} \mathrm{~h}^{-1}$ ) para o cálculo das faixas de suficiência na população estudada. A utilização de produtividades diferentes para o cálculo da chance matemática (4.000 e $\left.4.500 \mathrm{~kg} \mathrm{ha}^{-1}\right)$, na população estudada, não apresenta diferença entre as faixas de suficiência para os macronutrientes. As faixas encontradas para os macronutrientes com o método da chance matemática foram, em g kg-1, de 33-45; 3,8-5,3; 15-21; 20-36; 4,0-7,2 e 2,8-8,6 para N, $P, K, C a, M g$ e S, respectivamente. Os resultados obtidos não diferem de outros trabalhos, mas podem ser utilizados para uma região em específico.
\end{abstract}

Palavras-chave: avaliação nutricional, diagnose foliar, Gossypium hirsutum r. latifolium.

\section{ABSTRACT}

The cotton growth occupies about 1.1 million hectares in Brazil and the midwest, especially the state of Mato
Grosso, is the largest producer in the country. This work aimed to establish the sufficiency ranges for macronutrients in cotton crops by the method of mathematical chance. The work was carried out from 2000 to 2003 in the cities of Costa Rica (MS), Diamantino and Sapezal (MT), totaling 152 samples of plant tissue and yield. The chance mathematics method was adequate for to establish cotton macronutrients references values, can be to help parameters were the conventional research cannot to prove in the small time space. The use of different yields for the calculation of mathematical chance $\left(4,000\right.$ and $\left.4,500 \mathrm{~kg} \mathrm{ha}^{-1}\right)$ in the population studied, no difference between the sufficiency ranges for macronutrients. The macronutrients sufficiency range was, in the $\mathrm{mg} \mathrm{kg}^{-1}, 33-45 ; 3,8-5,3 ; 15-21 ; 20-36 ; 4,0-7,2$ e 2,8-8,6 for N, P, K, Ca, Mg e S, respectly. The indicated range, however to similar with the recommendations published, they demonstrate improvement about to obtainment of increases productivities.

Key words: nutritional available, leave diagnosis, sufficiency range, Gossypium hirsutum $r$. latifolium.

\section{INTRODUÇÃO}

A cotonicultura ocupa cerca de 1,1 milhão de hectares no Brasil segundo o IBGE. O Estado do Mato Grosso é o maior produtor do país, obtendo, na safra 2010/2011, um valor bruto da produção de algodão em torno de R \$ 4,9 bilhões. Essa cultura possui valores de referência para avaliação do estado nutricional (SILVA \& RAIJ, 1996; RIBEIRO et al., 1999; SOUSA \& LOBATO, 2004). Entretanto, a maioria desses valores é oriunda de trabalhos realizados nas décadas de 70 e 80

\footnotetext{
IUniversidade Estadual de Mato Grosso do Sul (UEMS), Campus de Aquidauana, Rod. Aquidauana/Piraputanga, km 12, CP 25, 79200-000, Aquidauana, MS, Brasil. E-mail: camacho@uems.br. Autor para correspondência.

IIFaculdade de Ciências Agrárias e Veterinárias (FCAV), Universidade Estadual Paulista “Júlio de Mesquita Filho” (UNESP),
} Jaboticabal, SP, Brasil. 
no Estado de São Paulo e obtidos segundo a filosofia do nível crítico. Essa tem aplicabilidade mais restrita às condições ambientais em que os dados são gerados, uma vez que há uma tendência geral de modificação do sistema produtivo, que foi adaptado e tecnificado no Brasil nas últimas duas décadas, aliando-se ao fato do intenso trabalho de melhoramento vegetal da cultura, com lançamento de novas variedades de diferentes bases genéticas (BELTRÃO et al., 2008).

$\mathrm{Na}$ avaliação do estado nutricional das plantas, estabelece-se uma comparação entre dados de uma amostra e respectivos valores de referência para a cultura. Esses valores são geralmente obtidos em experimentos de calibração de nutrientes, nos quais é possível controlar características genéticas e ambientais, tendo como único fator de variação o nutriente objeto de estudo (MALAVOLTA et al., 1997).

Valores-padrão podem ser estabelecidos a partir de dados obtidos em talhões comerciais, que se encontram sob as mais variadas condições ecofisiológicas, por meio do método da chance matemática (WADT et al., 1998). Na cultura do algodoeiro, alguns trabalhos têm sido desenvolvidos na área de diagnose foliar, como aqueles que visam ao estabelecimento de normas DRIS e CND (SILVA et al., 1999; SERRA et al., 2010a), que utilizam a avaliação de nutrientes aos pares ou variáveis multinutrientes. De acordo com SERRA et al. (2010b), estabelecendo faixas de suficiência para o algodoeiro, com dados oriundos da região oeste do Estado da Bahia, foi verificado que os valores das faixas foram idênticos pelos métodos DRIS e CND, enquanto o método da chance matemática retornou valores diferentes, embora similares aos dois outros métodos.

O objetivo do presente trabalho foi estabelecer faixas de suficiência para macronutrientes na cultura do algodoeiro por meio do método da chance matemática.

\section{MATERIAL E MÉTODOS}

As informações do monitoramento nutricional são oriundas de 152 áreas, nas quais foram coletadas amostras no período correspondente a três safras (2000/2001 a 2002/2003), oriundas de talhões comerciais dos municípios Costa Rica (Estado de Mato Grosso do Sul), Diamantino e Sapezal (Estado de Mato Grosso).

A distribuição das áreas avaliadas, bem como o detalhamento do manejo da adubação estão descritos detalhadamente em SILVA et al. (2009). O manejo da cultura, nos diferentes locais, foi realizado de acordo com as necessidades da lavoura, tais como aplicações de defensivos agrícolas (inseticidas, herbicidas e fungicidas), de adubos e de corretivos. A calagem foi realizada pelo método da saturação por bases, utilizando $\mathrm{V}_{2}=70 \%$, preconizado por SILVA \& RAIJ (1996), sendo a dose mínima usada de $0,8 \mathrm{Mg} \mathrm{ha}^{-1} \mathrm{e}$ a dose máxima de $5,5 \mathrm{Mg} \mathrm{ha}^{-1}$, sendo cada talhão manejado de maneira diferenciada em função da análise de solo.

Nas áreas avaliadas, foram realizadas amostragens de folhas durante o período de florescimento da cultura, que compreendeu os estádios fenológicos F2 a F4 (MARUR \& RUANO, 2001), com amostras compostas por folhas completas (limbo+pecíolo), sendo que as amostragens seguiram o que preconiza RIBEIRO et al. (1999), retiradas na quinta posição a partir do ápice, na haste principal. As amostras foram lavadas e secas em estufa com circulação forçada de $\operatorname{ar}\left(65^{\circ} \mathrm{C} \pm 5^{\circ} \mathrm{C}\right)$. Após a secagem, foram moídas e submetidas à determinação química dos nutrientes para avaliação dos teores foliares de nitrogênio $(\mathrm{N})$, fósforo $(\mathrm{P})$, potássio $(\mathrm{K})$, cálcio $(\mathrm{Ca})$, magnésio $(\mathrm{Mg})$ e enxofre $(\mathrm{S})$, conforme metodologia proposta por MALAVOLTA et al. (1997). A produtividade do algodoeiro, em $\mathrm{kg} \mathrm{ha}^{-1}$ de algodão em caroço, foi estimada no período da colheita com base na produtividade de cada área, por meio de colhedora mecanizada.

A análise pelo método da chance matemática foi realizada conforme indicações de WADT et al. (1998) para cada um dos nutrientes, utilizando-se duas relações, denominadas doravante de probabilidade 1 $\left(\mathrm{P}_{1}\right)$ e probabilidade $2\left(\mathrm{P}_{2}\right)$, sendo calculadas da seguinte maneira: $\mathrm{P}_{1}=\mathrm{A}_{\mathrm{i}}{ }^{*} \mathrm{~A}^{-1}$ e $\mathrm{P}_{2}=\mathrm{A}_{\mathrm{i}}{ }^{*} \mathrm{C}_{\mathrm{i}}^{-1}$, em que: $\mathrm{A}_{\mathrm{i}}=$ número de amostras com alta produtividade na classe $\mathrm{i}$; $\mathrm{A}=$ número total de amostras com alta produtividade; $\mathrm{C}_{\mathrm{i}}=$ número total de amostras na classe i. Para o cálculo do valor do índice de chance matemática (CHM), utilizase a seguinte fórmula: $\mathrm{CHM}_{\mathrm{Mi}}=\left[\left(\mathrm{P}_{1} * \mathrm{Pm}_{\mathrm{i}}\right) *\left(\mathrm{P}_{2} * \mathrm{Pm}_{\mathrm{i}}\right)\right]^{1 / 2}$, em que: $\mathrm{CHM}_{\mathrm{Mi}}$ é a chance matemática de um determinado nutriente $(\mathrm{M})$ pertencer à classe $\mathrm{i} ; \mathrm{P}_{1}$ e $\mathrm{P}_{2}$

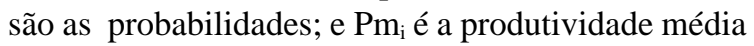
na classe $\mathrm{i}$.

A amplitude de classe foi definida, inicialmente, pela sugestão de WADT (1998), como sendo $A_{c}=A_{t}$. $n^{-1}$, em que $A_{c}=$ amplitude de classe; $A_{t}$ = amplitude total; e $\mathrm{n}=$ número de classes. $\mathrm{O}$ número de classes é estabelecido de acordo com a fórmula $\mathrm{n}=$ $\mathrm{N}^{1 / 2}$, em que $\mathrm{N}=$ número de observações. Entretanto, os resultados obtidos foram baseados em 12 classes, com amplitudes muito grandes, o que poderia resultar em inferências equivocadas, logo, os cálculos foram refeitos utilizando-se amplitudes de classes diferenciadas para cada nutriente, sendo que o número 
de classes foi função da razão entre a amplitude total e a amplitude de classe do nutriente estudado, resultando em número de classes diferentes, definidos em função da amplitude para cada nutriente, buscando melhorar os resultados de produtividade. A distribuição das classes por nutrientes é apresentada na tabela 1.

Para classificação dos talhões quanto à produtividade (alta ou baixa), foram utilizados os valores de 4000 e $4500 \mathrm{~kg} \mathrm{ha}^{-1}$, tendo como critério para o cálculo, aproximadamente, a média e a média mais um desvio padrão, respectivamente.

Após os cálculos dos respectivos valores da chance matemática para as populações de referência com produtividades $>4000$ e $>4500 \mathrm{~kg} \mathrm{ha}^{-1}\left(\mathrm{CHM}_{4000} \mathrm{e}\right.$ $\mathrm{CHM}_{4500}$ ), a escolha da faixa adequada foi realizada em função da proximidade dos maiores valores calculados para cada nutriente.

\section{RESULTADOS E DISCUSSÃO}

O maior valor encontrado para $\mathrm{CHM}_{4.000}$ foi de $1.485 \mathrm{~kg} \mathrm{ha}^{-1}$, sendo o maior valor correspondente à faixa ótima (WADT et al., 1998). Entretanto, se adotássemos esta faixa ótima como faixa de suficiência, teríamos a recomendação de 35 a $37 \mathrm{~g}$ de $\mathrm{N} \mathrm{kg}^{-1}$ de matéria seca na folha indicadora do algodão, que é muito estreita e, talvez, não correspondesse exatamente à prática associada à faixa de suficiência. Logo, como os maiores valores de chance matemática estavam agrupados (Tabela 2), todos os valores maiores que $1.200 \mathrm{~kg} \mathrm{ha}^{-1}$ foram considerados para a determinação da faixa de suficiência para expectativa de produtividade de $4.000 \mathrm{~kg} \mathrm{ha}^{-1}$ de algodão em caroço. Esse mesmo raciocínio foi utilizado para definição da faixa de suficiência com expectativa de produtividades maiores que $4.500 \mathrm{~kg} \mathrm{ha}^{-1}$ de algodão em caroço, já que as classes escolhidas para a composição da chance matemática foram aquelas que apresentaram valores de $\mathrm{CHM}_{4.500}$ maiores que $700 \mathrm{~kg} \mathrm{ha}^{-1}$.

Tabela 1 - Valores máximo e mínimo encontrados no banco de dados, amplitude escolhida de cada classe e número de classes utilizadas por nutriente avaliado.

\begin{tabular}{|c|c|c|c|c|}
\hline Nutriente & Máximo & Mínimo & $\begin{array}{l}\text { Amplitude } \\
\text { da classe }\end{array}$ & $\begin{array}{l}\text { Número } \\
\text { de classes }\end{array}$ \\
\hline \multicolumn{5}{|c|}{ 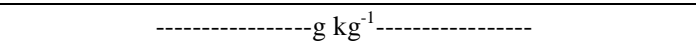 } \\
\hline $\mathrm{N}$ & 68,5 & 19,8 & 2 & 25 \\
\hline $\mathrm{P}$ & 5,3 & 2,1 & 0,2 & 16 \\
\hline $\mathrm{K}$ & 34,8 & 5,6 & 2 & 15 \\
\hline $\mathrm{Ca}$ & 43,8 & 8,0 & 2 & 18 \\
\hline $\mathrm{Mg}$ & 8,8 & 2,9 & 0,2 & 30 \\
\hline $\mathrm{S}$ & 8,6 & 2,1 & 0,2 & 33 \\
\hline
\end{tabular}

O agrupamento dos maiores valores encontrado pelo método da chance matemática indica maior probabilidade de resposta à adubação contendo o nutriente avaliado, além de eliminar os intervalos (de teores foliares do nutriente) que apresentaram baixas produtividades.

As faixas de suficiência para o teor de N (33 a $43 \mathrm{~g} \mathrm{~kg}^{-1}$ para a produtividade $4.000 \mathrm{~kg} \mathrm{ha}^{-1}$ e 33 a $45 \mathrm{~g}$ $\mathrm{kg}^{-1}$ para a produtividade $4.500 \mathrm{~kg} \mathrm{ha}^{-1}$ ) foram semelhantes a diversas recomendações existentes (SILVA, 1995; SILVA\& RAIJ, 1996; RIBEIRO et al., 1999; FUNDAÇÃO MT, 2001; OLIVEIRA, 2004) (Tabela 3). O método da chance matemática possui a vantagem de utilizar dados oriundos de talhões comerciais, mesmo na ausência de condições controladas, o que corrobora WADT (1998). Experimentos conduzidos no estado de São Paulo, na década de 1970, descritos por KIEHL et al. (1985), apresentaram maiores produtividades do algodoeiro quando os teores foliares de $\mathrm{N}$ variavam entre 30 e $44 \mathrm{~g}$ de $\mathrm{N} \mathrm{kg}^{-1}$, resultado semelhante ao observado neste trabalho. SABINO et al. (1994) indicaram uma faixa de 41 a $48 \mathrm{~g} \mathrm{de} \mathrm{N} \mathrm{kg}^{-1}$ e OLIVEIRA (1994), estudando doses de $\mathrm{N}$ e adubos verdes, verificaram que os teores de $\mathrm{N}$ adequados à cultura do algodoeiro encontravam-se entre 38 a $42 \mathrm{~g}$ de $\mathrm{N} \mathrm{kg}^{-1}$.

Para a escolha da faixa de suficiência de $\mathrm{P}$ para a cultura do algodoeiro, foram agrupados valores de CHM maiores que $700 \mathrm{~kg} \mathrm{ha}^{-1}$ no $\mathrm{CHM}_{4.000}$ e maiores que $800 \mathrm{~kg} \mathrm{ha}^{-1}$ no $\mathrm{CHM}_{4.500}$, retornando valores de referência elevados (2,6 a 5,0 e 3,8 a 5,3g kg ) (Tabela 2), próximos aos apresentados por SILVA \& RAIJ (1996), FUNDAÇÃO MT (2001) e OLIVEIRA (2004), muito embora o limite superior $\left(5,3 \mathrm{~g} \mathrm{~kg}^{-1}\right)$ tenha sido maior que várias referências consultadas (Tabela 3).

Os valores encontrados foram superiores aos indicados por ROSOLEM et al. (2000), que recomendaram concentrações foliares de $P$ entre 2,3 e $2,6 \mathrm{~g} \mathrm{~kg}^{-1}$. O maior valor de referência para o $\mathrm{P}$, observado neste trabalho, está relacionado às novas variedades de algodão disponíveis no mercado, já que se espera uma maior demanda pelo nutriente à medida que a produtividade é aumentada, com reflexo nos teores foliares ótimos dependentes da eficiência da planta na utilização do nutriente. A variabilidade na capacidade e eficiência da planta em absorver P do solo (EPSTEIN \& BLOOM, 2004) é uma característica varietal que pode ter afetado os valores críticos dos teores foliares observados.

O K foi agrupado pelos valores de CHM maiores que 600 e $1.000 \mathrm{~kg} \mathrm{ha}^{-1}$, para as produtividades de 4.000 e $4.500 \mathrm{~kg} \mathrm{ha}^{-1}$, respectivamente (Tabela 2). As faixas encontradas (11 a 25 e 15 a $21 \mathrm{~g} \mathrm{~kg}^{-1}$ ) foram compatíveis com outras recomendações (SILVA \& 
Tabela 2 - Distribuição das classes e valores da chance matemática das classes utilizadas para o N, P e K

\begin{tabular}{|c|c|c|c|c|c|c|c|c|}
\hline Classe & $\mathrm{CHM}_{4000}$ & $\mathrm{CHM}_{4500}$ & Classe & $\mathrm{CHM}_{4000}$ & $\mathrm{CHM}_{4500}$ & Classe & $\mathrm{CHM}_{4000}$ & $\mathrm{CHM}_{4500}$ \\
\hline$g k^{-1}$ & & & $g \mathrm{~kg}^{-1}$ & & & $g k^{-1}$ & & \\
\hline$=21$ & 418 & 0 & $=2,2$ & 231 & 0 & $=7$ & 435 & 0 \\
\hline $21: 23$ & 210 & 0 & $2,2: 2,4$ & 307 & 0 & $7: 9$ & 278 & 0 \\
\hline $23: 25$ & 335 & 0 & $2,4: 2,6$ & 449 & 493 & $9: 11$ & 468 & 0 \\
\hline $25: 27$ & 0 & 0 & $\begin{array}{l:l}2,6 & 2,8\end{array}$ & 743 & 245 & $11: 13$ & 988 & 0 \\
\hline $\begin{array}{l:l}27 & 29\end{array}$ & 0 & 0 & $\begin{array}{l:l}2,8 & 3,0\end{array}$ & 1314 & 648 & $13: 15$ & 982 & 359 \\
\hline $29: 31$ & 672 & 368 & $3,0: 3,2$ & 563 & 0 & $15: 17$ & 1483 & 1220 \\
\hline $31: 33$ & 675 & 370 & $3,2: 3,4$ & 907 & 560 & $17: 19$ & 1558 & 1658 \\
\hline $33: 35$ & 1309 & 718 & $3,4: 3,6$ & 359 & 0 & $19: 21$ & 1456 & 1065 \\
\hline $35: 37$ & 1485 & 752 & $\begin{array}{l:l}3,6 & 3,8\end{array}$ & 390 & 0 & $21: 23$ & 704 & 0 \\
\hline $37: 39$ & 1243 & 909 & $\begin{array}{l:l}3,8 & 4,0\end{array}$ & 955 & 873 & $23: 25$ & 682 & 561 \\
\hline $39: 41$ & 1301 & 713 & $\begin{array}{l:l}4,0 & 4,2\end{array}$ & 1550 & 911 & $25: 27$ & 377 & 414 \\
\hline $41: 43$ & 1381 & 1363 & $4,2: 4,4$ & 1304 & 1191 & $27: 29$ & 366 & 0 \\
\hline $43: 45$ & 675 & 740 & $4,4: 4,6$ & 1015 & 835 & $\begin{array}{l:l}29 & 31\end{array}$ & 269 & 0 \\
\hline $45: 47$ & 680 & 480 & $4,6: 4,8$ & 721 & 593 & $31: 33$ & 0 & 0 \\
\hline $47: 49$ & 298 & 0 & $4,8: 5,0$ & 788 & 648 & $>33$ & 0 & 0 \\
\hline $49: 51$ & 387 & 0 & $>5,0$ & 515 & 847 & - & - & - \\
\hline $51: 53$ & 552 & 227 & - & - & - & - & - & - \\
\hline $53: 55$ & 558 & 0 & - & - & - & - & - & - \\
\hline $55: 57$ & 628 & 258 & - & - & - & - & - & - \\
\hline $57: 59$ & 0 & 0 & - & - & - & - & - & - \\
\hline $59: 61$ & 402 & 0 & - & - & - & - & - & - \\
\hline $61: 63$ & 210 & 0 & - & - & - & - & - & - \\
\hline $63: 65$ & 282 & 0 & - & - & - & - & - & - \\
\hline $65: 67$ & 178 & 0 & - & - & - & - & - & - \\
\hline$>67$ & 286 & 0 & - & - & - & - & - & - \\
\hline
\end{tabular}

RAIJ, 1996; RIBEIRO et al., 1999; FUNDAÇÃO MT, 2001; OLIVEIRA, 2004). ROSOLEM \& MACHADO (1984) consideraram adequados teores de $\mathrm{K}$ de 13,3 a $15,9 \mathrm{~g} \mathrm{~kg}^{-1}$. Observa-se que o método da chance matemática indicou valores superiores que corroboram,

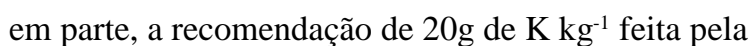
FUNDAÇÃO MT (2001), a qual publica resultados direcionados ao estado de Mato Grosso. Esse valor está dentro da faixa de suficiência apresentada pelo método da chance matemática.

Tabela 3 - Valores de referência de macronutrientes na cultura do algodoeiro

\begin{tabular}{|c|c|c|c|c|c|c|}
\hline & $\mathrm{N}$ & $\mathrm{P}$ & $\mathrm{K}$ & $\mathrm{Ca}$ & $\mathrm{Mg}$ & S \\
\hline $\mathrm{CHM}_{4000}{ }^{1}$ & $33-43$ & $2,6-5,0$ & $11-25$ & $16-36$ & $3,0-7,2$ & $2,8-8,0$ \\
\hline $\mathrm{CHM}_{4500}{ }^{1}$ & $33-45$ & $3,8-5,3$ & $15-21$ & $20-36$ & $4,0-7,2$ & $2,8-8,6$ \\
\hline SILVA $(1995)^{1}$ & 35 & 2,5 & 15 & 20 & 3 & 4 \\
\hline RIBEIRO et al. (1999) ${ }^{1}$ & 32 & 1,7 & 15 & 20 & 5 & 4 \\
\hline SILVA \& RAIJ (1996) ${ }^{2}$ & $35-43$ & $2,5-4,0$ & $15-25$ & $20-35$ & $3-8$ & $4-8$ \\
\hline FUNDAÇÃO MT (2001)² & $40-45$ & 3,2 & 20 & - & - & - \\
\hline OLIVEIRA $(2004)^{2}$ & $35-40$ & $2-4$ & $15-25$ & $20-35$ & $3-8$ & $4-8$ \\
\hline MALAVOLTA et al. $(1997)^{3}$ & $35-40$ & $2-2,5$ & $14-16$ & $30-40$ & $4-5$ & $2-3$ \\
\hline YAMADA \& LOPES $(1998)^{3}$ & - & - & - & $25-35$ & $4-8$ & $4-6$ \\
\hline
\end{tabular}

${ }^{1}$ Folha completa (limbo+pecíolo); ${ }^{2}$ Limbo foliar; ${ }^{3}$ Não identificado. 
A escolha da faixa de suficiência para o Ca e o Mg (Tabela 4) compreenderam classes quando os valores de $\mathrm{CHM}_{4.000}$ foram superiores a $700 \mathrm{~kg} \mathrm{ha}^{-1}$ enquanto para o $\mathrm{CHM}_{4.500}$ foram utilizados valores superiores a $600 \mathrm{~kg} \mathrm{ha}^{-1}$.

A escolha da faixa adequada para o $\mathrm{Ca}$, a exemplo do P, compreende valores de CHM menores dos que os utilizados para a separação das classes (Tabela 4). Os valores utilizados para $\mathrm{CHM}_{4000}$ foram aqueles superiores a $700 \mathrm{~kg} \mathrm{ha}^{-1}$, enquanto para o $\mathrm{CHM}_{4500}$ foram utilizados valores superiores a $600 \mathrm{~kg}$ ha $^{-1}$. Essa dispersão dos valores de CHM também foi observada para o Mg (Tabela 4), porém, a falta de agrupamento dos maiores valores em classes próximas dificultou a escolha da faixa adequada. Logo, para $\mathrm{CHM}_{4000}$, foram incorporadas todas as classes até 7,2g de $\mathrm{Mg} \mathrm{kg}{ }^{-1}$, visto que não foi possível agrupar os dados. Esse fato pode refletir que, na realidade, este nutriente não foi o fator limitante nas áreas avaliadas. Mais uma vez, é levantada hipótese de que provavelmente o Mg não tenha sido limitante em nenhuma das áreas avaliadas, devido à ocorrência de altos valores de CHM em classes distantes uma das outras (Tabela 4). Essa hipótese pode, também, ser aplicada no caso do S (Tabela 4), em que os valores de chance matemática foram dispersos.

Tabela 4 - Distribuição das classes e valores da chance matemática das classes utilizadas para o Ca, Mg e S.

\begin{tabular}{|c|c|c|c|c|c|c|c|c|}
\hline Classe & $\mathrm{CHM}_{4000}$ & $\mathrm{CHM}_{4500}$ & Classe & $\mathrm{CHM}_{4000}$ & $\mathrm{CHM}_{4500}$ & Classe & $\mathrm{CHM}_{4000}$ & $\mathrm{CHM}_{4500}$ \\
\hline$g k g^{-1}$ & & & $g \mathrm{~kg}^{-1}$ & & & $g \mathrm{~kg}^{-1}$ & & \\
\hline$=10$ & 441 & 0 & $=3,0$ & 881 & 0 & $=2,2$ & 260 & 0 \\
\hline $10: 12$ & 230 & 0 & $3,0: 3,2$ & 613 & 0 & $\begin{array}{l:l}2,2 & 2,4\end{array}$ & 472 & 0 \\
\hline $12: 14$ & 425 & 0 & $3,2: 3,4$ & 0 & 0 & $\begin{array}{l:l}2,4 & 2,6\end{array}$ & 668 & 0 \\
\hline $14: 16$ & 572 & 0 & $3,4: 3,6$ & 489 & 0 & $\begin{array}{l:l}2,6 & 2,8\end{array}$ & 144 & 0 \\
\hline $16: 18$ & 782 & 184 & $3,6: 3,8$ & 235 & 0 & $2,8: 3,0$ & 883 & 1163 \\
\hline $18: 20$ & 1050 & 173 & $3,8: 4,0$ & 0 & 0 & $\begin{array}{l:l}3,0 & 3,2\end{array}$ & 1240 & 556 \\
\hline $20: 22$ & 717 & 674 & $\begin{array}{l:l}4,0 & 4,2\end{array}$ & 713 & 703 & $3,2: 3,4$ & 1085 & 595 \\
\hline $22: 24$ & 348 & 0 & $4,2: 4,4$ & 820 & 270 & $\begin{array}{l:l}3,4 & 3,6\end{array}$ & 792 & 652 \\
\hline $24: 26$ & 434 & 178 & $4,4: 4,6$ & 1123 & 924 & $\begin{array}{l:l}3,6 & 3,8\end{array}$ & 810 & 148 \\
\hline $26: 28$ & 1261 & 1244 & $4,6: 4,8$ & 687 & 226 & $\begin{array}{l:l}3,8 & 4,0\end{array}$ & 469 & 0 \\
\hline $28: 30$ & 1654 & 1632 & $4,8: 5,0$ & 691 & 227 & $\begin{array}{l:l}4,0 & 4,2\end{array}$ & 534 & 220 \\
\hline $\begin{array}{l:l}30 & 32\end{array}$ & 1257 & 752 & $\begin{array}{l:l}5,0 & 5,2\end{array}$ & 433 & 356 & $\begin{array}{l:l}4,2 & 4,4\end{array}$ & 372 & 612 \\
\hline $32: 34$ & 1071 & 1007 & 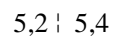 & 1255 & 688 & $\begin{array}{l:l}4,4 & 4,6\end{array}$ & 0 & 0 \\
\hline $34: 36$ & 1174 & 644 & $5,4: 5,6$ & 987 & 443 & $\begin{array}{l:l}4,6 & 4,8\end{array}$ & 632 & 520 \\
\hline $36: 38$ & 0 & 0 & $5,6: 5,8$ & 423 & 232 & $\begin{array}{l:l}4,8 & 5,0\end{array}$ & 703 & 578 \\
\hline $38: 40$ & 296 & 487 & $\begin{array}{l:l}5,8 & 6,0\end{array}$ & 563 & 232 & $\begin{array}{l:l}5,0 & 5,2\end{array}$ & 785 & 323 \\
\hline $40: 42$ & -- & -- & $6,0: 6,2$ & 1035 & 1064 & $\begin{array}{l:l}5,2 & 5,4\end{array}$ & 592 & 325 \\
\hline$>42$ & 0 & 0 & $6,2: 6,4$ & 811 & 1001 & $\begin{array}{l:l}5,4 & 5,6\end{array}$ & 627 & 258 \\
\hline - & - & - & $6,4: 6,6$ & 676 & 1111 & $\begin{array}{l:l}5,6 & 5,8\end{array}$ & 317 & 521 \\
\hline - & - & - & $6,6: 6,8$ & 680 & 373 & $\begin{array}{l:l}5,8 & 6,0\end{array}$ & 0 & 0 \\
\hline - & - & - & $6,8: 7,0$ & 657 & 540 & $\begin{array}{l:l}6,0 & 6,2\end{array}$ & 427 & 0 \\
\hline- & - & - & $\begin{array}{l:l}7,0 & 7,2\end{array}$ & 710 & 1169 & $\begin{array}{l:l}6,2 & 6,4\end{array}$ & 471 & 775 \\
\hline- & - & - & $7,2: 7,4$ & -- & -- & $6,4: 6,6$ & -- & -- \\
\hline- & - & - & $7,4: 7,6$ & -- & -- & $6,6: 6,8$ & 527 & 868 \\
\hline- & - & - & $7,6: 7,8$ & -- & -- & $6,8: 7,0$ & 574 & 315 \\
\hline- & - & - & $7,8: 8,0$ & 0 & 0 & $\begin{array}{l:l}7,0 & 7,2\end{array}$ & 639 & 526 \\
\hline - & - & - & $\begin{array}{l:l}8,0 & 8,2\end{array}$ & -- & -- & $7,2: 7,4$ & 694 & 761 \\
\hline - & - & - & $8,2: 8,4$ & -- & -- & $\begin{array}{l:l}7,4 & 7,6\end{array}$ & -- & -- \\
\hline - & - & - & $8,4: 8,6$ & -- & -- & $\begin{array}{l:l}7,6 & 7,8\end{array}$ & 522 & 859 \\
\hline- & - & - & $>8,6$ & 0 & 0 & $7,8: 8,0$ & 843 & 925 \\
\hline - & - & - & - & - & - & $\begin{array}{l:l}8,0 & 8,2\end{array}$ & -- & -- \\
\hline - & - & - & - & - & - & $8,2: 8,4$ & -- & -- \\
\hline- & - & - & - & - & - & $>8,4$ & 473 & 779 \\
\hline
\end{tabular}


Ao se comparar os resultados obtidos para Ca e Mg com o trabalho de ROSOLEM \& MACHADO (1984), evidencia-se que os teores apresentados por estes autores são menores para Ca (35,6 a 38,3g kg-1) e coincidentes para $\mathrm{Mg}\left(5,3 \mathrm{a} 5,8 \mathrm{~g} \mathrm{~kg}^{-1}\right)$. De maneira geral, os valores de Ca e Mg encontrados pelo método da chance matemática estiveram de acordo com os padrões estabelecidos para a cultura (SILVA \&RAIJ, 1996; YAMADA \& LOPES, 1998; RIBEIRO et al., 1999; OLIVEIRA, 2004) (Tabela 3).

As faixas de suficiência estabelecidas para o S (Tabela 3) foram similares às recomendadas por alguns padrões estabelecidos (SILVA \&RAIJ, 1996; YAMADA \& LOPES, 1998; OLIVEIRA, 2004), possivelmente o que indica um suprimento adequado deste nutriente dentro da população estudada. Para o $\mathrm{S}$, bem como para todos os outros macronutrientes $(\mathrm{N}$, P, K, Ca e Mg), a separação das classes de produtividade (4.000 e $4.500 \mathrm{~kg} \mathrm{ha}^{-1}$ de algodão em caroço) não apresentaram diferenciação, o que indica uma sobreposição das faixas estimadas, e que poderíamos unificar ambas as faixas.

\section{CONCLUSÃO}

A utilização de produtividades diferentes para o cálculo da chance matemática (4.000 e 4.500kg ha $\left.{ }^{-1}\right)$, na população estudada, não apresenta diferença entre as faixas de suficiência para os macronutrientes. As faixas encontradas para os macronutrientes com o método da chance matemática foram, em $\mathrm{g} \mathrm{kg}^{-1}$, de 3345; 3,8-5,3; 15-21; 20-36; 4,0-7,2 e 2,8-8,6 para N, P, K, $\mathrm{Ca}, \mathrm{Mg}$ e $\mathrm{S}$, respectivamente. Os resultados obtidos não diferem de outros trabalhos, mas podem ser utilizados para uma região específica.

\section{REFERÊNCIAS}

BELTRÃO, N.E.M; AZEVEDO, D.M.P (Eds.) O agronegócio do algodão no Brasil. Brasília: Embrapa Informação Tecnológica, 2008. 2v. 1.309p.

EPSTEIN, E; BLOOM, A.J. Nutrição mineral das plantas: princípios e perspectivas. Londrina: Editora Planta, 2004. 403p.

FUNDAÇÃO MT. Boletim de pesquisa de algodão. Rondonópolis, 2001. 238p. (Boletim, 4).

KIEHL, J.C. et al. Efeitos de doses e modos de aplicação de uréia na produção de algodão. Revista Brasileira de Ciência do Solo, Campinas, v.9, p.39-44, 1985.

MALAVOLTA, E. et al. Avaliação do estado nutricional das plantas: princípios e aplicações. 2.ed. Piracicaba: POTAFOS, 1997. 319p.

MARUR, C.J.; RUANO, O. A reference system for determination of developmental stages of upland cotton. Revista Oleaginosas e Fibrosas, Campina Grande, v.5, n.2, p.313-317, 2001.
OLIVEIRA, E.L. Coberturas verdes de inverno e adubação nitrogenada em algodoeiro. Revista Brasileira de Ciencia do Solo, Campinas, v.18, p.235-241, 1994.

OLIVEIRA, S.A. Análise foliar. In: SOUSA, D.M.G.; LOBATO, E. (Ed.). Cerrado: correção do solo e adubação. Brasília: Embrapa Informação Tecnológica, 2004. p.245-256.

RIBEIRO, A.C. et al. Recomendações para o uso de corretivos e fertilizantes em Minas Gerais - $5^{\mathrm{a}}$ aproximação. Viçosa, MG: UFV, 1999. 359p.

ROSOLEM, C.A. et al. Crescimento radicular e nutrição de cultivares de algodoeiro em resposta à calagem. Pesquisa Agropecuária Brasileira, Brasília, v.35, n.4, p.827-833, 2000. Disponível em: $<$ http://www.scielo.br/scielo.php?script=sci_arttext\&pid=S0100204X2000000400021\&lng=pt\&nrm=iso >. Acesso em: 30 jun. 2011. doi: 10.1590/S0100-204X2000000400021.

ROSOLEM, C.A.; MACHADO, J.R. Efeitos da calagem e gessagem na produção de algodão e na lixiviação de bases em dois latossolos. Revista Brasileira de Ciência do Solo, Campinas, v.8, p.103-109, 1984.

SABINO, J.C. et al. Aplicação de uréia em cobertura e via foliar na cultura do algodoeiro. Revista Brasileira de Ciência do Solo, Campinas, v.18, p.477-482, 1994.

SERRA, A.P. et al. Desenvolvimento de normas DRIS e CND e avaliação do estado nutricional da cultura do algodoeiro. Revista Brasileira de Ciência do Solo, Viçosa, v.34, p.97104, 2010a. Disponível em: <http://dx.doi.org/10.1590/S010006832010000100011>. Acesso em: 30 jun. 2011.

SERRA, A.P. et al. Determinação de faixas normais de nutrientes no algodoeiro pelos métodos CHM, DRIS e CND. Revista Brasileira de Ciência do Solo, Viçosa, v.34, p.105-113, 2010a. Disponível em: <http://dx.doi.org/10.1590/S010006832010000100010>. Acesso em: 30 jun. 2011.

SILVA, M.A.C. et al. Estabelecimento e validação de normas DRIS para a cultura do algodão no centro-oeste do Brasil. Acta Scientiarium Agronomy, Maringá, v.31, n.1, p.93-99, 2009. Disponível em: <http://periodicos.uem.br/ojs/index.php/ ActaSciAgron/article/view/6636/6636>. Acesso em: 30 jun. 2011. doi: 10.4025/actasciagron.v31i1.6636.

SILVA, N.M.; RAIJ, B. Fibrosas. In: RAIJ, B. et al. Recomendação de adubação e calagem para o Estado de São Paulo. Campinas: Instituto Agronômico/Fundação IAC, 1996. 285p. (Boletim técnico, 100).

SILVA, N.M. et al. Seja o doutor do seu algodoeiro. Piracicaba: Potafos, 1995. 24p. (Arquivo do Agrônomo, 8).

SOUSA, D.M.G.; LOBATO, E. (Ed.). Cerrado: correção do solo e adubação. Brasília: Embrapa Informação Tecnológica, 2004. 416p.

WADT, P.G.S. et al. O método da chance matemática na interpretação de dados de levantamento nutricional de eucalipto. Revista Brasileira de Ciência do Solo, Viçosa, v.22, p.773778, 1998.

YAMADA, T.; LOPES, A.S. Balanço de nutrientes na agricultura brasileira. Piracicaba: POTAFOS, 1998. 8p. (Encarte Técnico, 84). 